F. Camilo and B. M. Gaensler, eds.

\title{
An X-Ray Pulsar, Metal-rich Ejecta, and Shocked Ambient Medium in the Supernova Remnant G292.0+1.8
}

\author{
John P. Hughes, Robert B. Friedman \\ Rutgers University, NJ, USA \\ Patrick Slane \\ Harvard-Smithsonian Center for Astrophysics, MA, USA \\ Sangwook Park \\ Pennsylvania State University, PA, USA
}

\begin{abstract}
We report the discovery of pulsed X-ray emission from the compact object CXOU J112439.1-591620 within the Galactic supernova remnant G292.0+1.8 using the High Resolution Camera on the Chandra $X$-Ray Observatory. The X-ray period is consistent with the extrapolation of the radio period and spindown rate of PSR J1124-5916. The X-ray pulse is single peaked and broad. There is no optical counterpart to a limit of $M_{V} \sim 26$. The pressure in the pulsar wind nebula is considerably less than that in the reverse-shock-heated ejecta and circumstellar medium, indicating that the reverse shock has not yet begun to interact with the nebula.
\end{abstract}

\section{Introduction}

G292.0+1.8 is a young, oxygen-rich supernova remnant (SNR) in the southern sky. The recent discovery of a pulsar wind nebula (PWN) (Hughes et al. 2001) and 135-ms period radio pulsar (Camilo et al. 2002) have greatly increased interest in this object, which, because of its youth, ejecta-dominated nature, and relative closeness, is sometimes considered the southern analog of Cassiopeia A. Here we report on the nature of the pulsar and discuss the evolutionary state of the SNR and PWN, especially as regards their interaction.

\section{X-Ray Pulsar}

The compact X-ray source CXOU J112439.1-591620 in G292.0+1.8 was observed with the Chandra high resolution camera (HRC) in timing mode for 50 ks on 2001 Jul 14. No pulse was detected in a coherent FFT of the entire light curve for the events (1324 in total) extracted from within a $2^{\prime \prime}$ radius of the point source. A search at pulse frequencies near the radio parameters yielded a significant detection of an X-ray pulsar (Fig. 1 left), confirming that the radio 

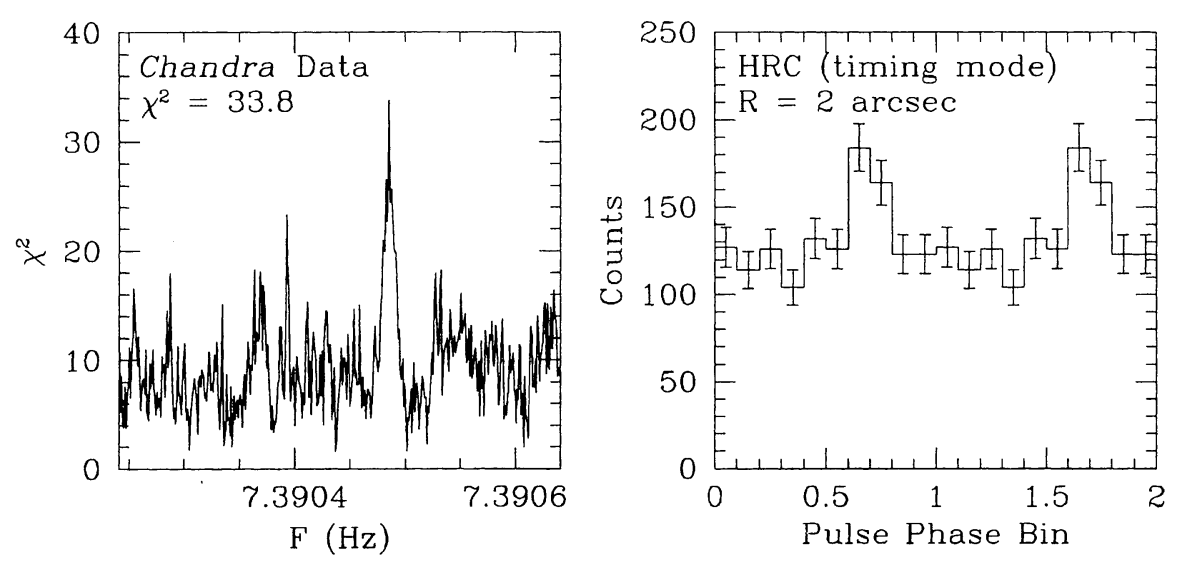

Figure 1. Left panel: $\chi^{2}$ vs. trial frequency for the compact central $\mathrm{X}$-ray source in G292.0+1.8. The peak frequency is consistent with that of PSR J1124-5916. Right panel: Pulse phase X-ray light curve.

pulsar, X-ray pulsar, and compact central X-ray source are one and the same. Agreement between the ages of the pulsar and SNR make a virtually ironclad case for an association between PSR J1124-5916 and SNR G292.0+1.8.

The X-ray pulse is single peaked and broad (Fig. 1 right). Of the total number of events in the light curve, only about 130 are pulsed. The X-ray image in the vicinity of the pulsar is complex: in addition to an unresolved source there is a small, extended, elliptically-shaped nebula. In fact the nebula dominates the flux from this region; only approximately 160 events (time-averaged) come from the unresolved pulsar. Comparing the pulsed to the time-averaged flux indicates that the X-ray pulsar is highly pulsed, $>65 \%$. A more complete report on the properties of the X-ray pulsar and its compact nebula can be found in Hughes et al. (2003).

\section{Optical Limits on PSR J1124-5916}

In a search for an optical counterpart, the pulsar was observed at the CTIO Blanco 4-m telescope with the MOSAIC II camera on 2002 Apr 17. Exposures were taken in one narrow-band $\mathrm{H} \alpha$ and four broadband filters for approximately 1 hour each. Standard reductions were carried out and the images were registered to the celestial sphere. No optical counterpart was detected (Fig. 2 left) down to limits in $B, V, R$ and $I$ of $26.8,26.4,26.2$, and 25.5 , respectively. The intrinsic optical luminosity for PSR J1124-5916, accounting for distance and extinction, is much below the luminosities of the three young pulsars Crab, PSR B0540-69 and PSR B1509-58, although the limit is still above the optical luminosity of PSR B0833-45 in Vela. In terms of the efficiency for converting spin down energy into optical luminosity, these new limits for PSR J1124-5916 imply a rather low value: $\epsilon=L_{V} / \dot{E}<1.7 \times 10^{-7}$. Again this falls far below the values 

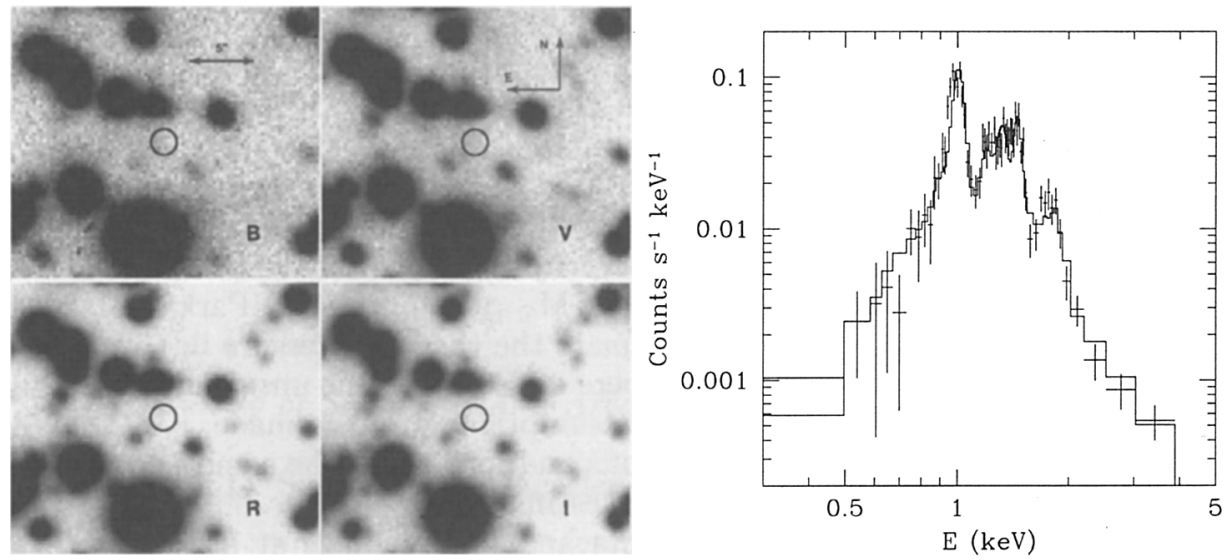

Figure 2. Left panel: Broadband optical images in the vicinity of PSR J1124-5916, whose position is denoted by the circle. North is up; east is to the left. Right panel: Chandra ACIS-S spectrum of an ejecta knot.

found for the three young isolated pulsars in SNRs, but is an order of magnitude or so higher than the value of $\epsilon$ for the Vela pulsar (Caraveo, Mereghetti \& Bignami 1994).

\section{Dynamical State of the PWN and SNR}

One of the most important issues regarding the evolutionary state of G292.0+1.8 concerns the relationship between the PWN, the free-streaming ejecta, and the reverse shock. By examining the pressures in the various regions of the remnant we have obtained some insight into this question.

The pressure in the pulsar wind nebula has been estimated in two rather different ways (Hughes et al. 2003): using the location of the pulsar termination shock and from the minimum energy condition applied to the radio synchrotron emission. Values obtained were $1.3 \times 10^{-10}$ dyne $\mathrm{cm}^{-2}$ (minimum energy) and $2.6 \times 10^{-9}$ dyne $\mathrm{cm}^{-2}$ (termination shock). Although it is possible that the pressure is as high as the latter value, in practice this would require the nebula to be almost entirely particle dominated with an energy content 40 times that of the minimum energy condition. Any value of pressure much higher than this would be very difficult to accommodate given what we currently know about the age and energetics of G292.0+1.8. It therefore seems reasonable to us to conclude that the true pressure in the PWN lies at or below $\sim 10^{-9}$ dyne $\mathrm{cm}^{-2}$.

Recently, a study of the thermal properties of G292.0+1.8 has been carried out by Park et al. (2004). Regions of normal composition, corresponding to shocked interstellar or circumstellar medium (ISM/CSM), were identified based on near-solar fitted abundance ratios. Using the densities and temperatures derived from the spectral fits for these regions, an emission geometry, and the 
assumption of equal electron and ion temperatures, an estimate for the thermal pressure of $8.8 \times 10^{-8}$ dyne $\mathrm{cm}^{-2}$ was obtained. There are numerous knots of X-ray emitting ejecta throughout the remnant. Figure 2 (right) shows the Chandra ACIS-S spectrum of one such knot located slightly south of the pulsar. The knot shows strong emission lines of $\mathrm{Ne}(\sim 1 \mathrm{keV}), \mathrm{Mg}(\sim 1.3 \mathrm{keV})$, and $\mathrm{Si}$ $(\sim 1.8 \mathrm{keV})$. The spectrum can be fully described with emission contributions from only these three species and oxygen; the data do not require the presence of any other elemental species. We identify this as a knot of pure ejecta from the hydrostatic burning layers of the $\sim 25 \mathrm{M}_{\odot}$ progenitor star (Park et al. 2004). In a similar manner to before we estimate the thermal pressure in this knot to be $5.9 \times 10^{-9}$ dyne $\mathrm{cm}^{-2}$. Although there are considerable uncertainties in these pressure estimates, the most critical assumptions we have made, e.g., that the plasma is unclumped and that electrons and ions share the same temperature, would tend to result in higher pressure estimates if relaxed.

The pressure in the shocked ejecta and ISM/CSM is at least a factor of two and more likely one to two orders of magnitude higher than the pressure in the PWN. This is strong evidence that the reverse shock has not yet begun to encounter the PWN. Furthermore, our ISM/CSM, ejecta, and PWN pressures are in remarkably good numerical agreement with dynamical models for a PWN interacting with freely expanding ejecta (see Fig. 4 of van der Swaluw et al. 2001). Finding evidence for the interaction between the PWN and the free streaming ejecta would be an important confirmation of this picture.

There should also be a reservoir of cold unshocked ejecta between the PWN and the reverse shock. One way to detect and investigate the composition of this material would be through absorption line studies of, for example, cold $\mathrm{Fe}$ or Si against the hard continuum emission of the PWN. This may be possible to do using Astro-E2 and should become routine with future higher sensitivity missions like the Constellation-X Facility.

Acknowledgments. The authors thank F. Camilo, B. Gaensler, M. Juda, P. Ghavamian, D. Burrows, J. Nousek, G. Garmire, and F. Winkler for help with various aspects of this research or for contributions to the presentation. Financial support was partially provided by Chandra grant GO1-2052X.

\section{References}

Camilo, F., Manchester, R. N., Gaensler, B. M., Lorimer, D. R., \& Sarkissian, J. 2002, ApJ, 567, L71

Caraveo, P. A., Mereghetti, S., \& Bignami, G. F. 1994, ApJ, 423, L125

Hughes, J. P., Slane, P. O., Burrows, D. N., Garmire, G., Nousek, J. A., Olbert, C. M., \& Keohane, J. W. 2001, ApJ, 559, L153

Hughes, J. P., Slane, P. O., Park, S., Roming, P. W. A., \& Burrows, D. N. 2003, ApJ, 591, L139

Park, S., Hughes, J. P., Slane, P. O., Burrows, D. N., Roming, P. W. A., Nousek, J. A., \& Garmire, G. P. 2004, ApJ, 602, L33

van der Swaluw, E., Achterberg, A., Gallant, Y. A., \& Tóth, G. 2001, A\&A, 380,309 